\title{
BMJ Open Parent-child conversations associated with alcohol-related risk behaviours in young people (13-17 years) in the UK: a cross-sectional study
}

\author{
Anna-Marie Jones (D) , ${ }^{1}$ Alexandra Sawyer, ${ }^{2}$ Jörg W Huber, ${ }^{2}$ Lester Coleman, ${ }^{2}$ \\ Nina Dunne, ${ }^{2}$ Nigel Sherriff ${ }^{2}$
}

To cite: Jones A-M, Sawyer A, Huber JW, et al. Parent-child conversations associated with alcohol-related risk behaviours in young people (13-17 years) in the UK: a crosssectional study. BMJ Open 2020;10:e033171. doi:10.1136/ bmjopen-2019-033171

- Prepublication history for this paper is available online. To view these files, please visit the journal online (http://dx.doi. org/10.1136/bmjopen-2019033171).

Received 24 July 2019 Revised 29 April 2020 Accepted 15 May 2020

Check for updates

(C) Author(s) (or their employer(s)) 2020. Re-use permitted under CC BY-NC. No commercial re-use. See rights and permissions. Published by BMJ.

${ }^{1}$ Research and Development, Sussex Partnership NHS Foundation Trust, Hove, UK ${ }^{2}$ School of Health Sciences, University of Brighton, Brighton, UK

Correspondence to Anna-Marie Jones; anna-marie.jones@ sussexpartnership.nhs.uk

\section{ABSTRACT}

Objective To investigate different types of parent-child conversations associated with young people's (13-17 years) alcohol-related risk behaviours.

Design Secondary analysis of the 2016 Drinkaware Monitor Survey. This survey employed a cross-sectional design and collected data using self-completion questionnaires.

Setting UK-wide.

Participants 561 parent-child pairs were included in the analysis. The nationally representative quota sample was weighted by reference to the UK population.

Methodology Data were analysed using purposeful selection modelling (adjusted OR (AOR), 95\% Cls).

Risk behaviours 'Whether have ever drank' and 'whether vomited as a result of alcohol'.

Results 50\% (277/553) of young people reported drinking a whole alcoholic drink, and $22 \%(60 / 277)$ of these experienced vomiting as a result. After adjusting for age and gender, the likelihood of ever having drank alcohol was significantly increased among the following young people: those whose parents believed they knew a little about how much they drink (AOR 1.80, 95\% Cl 1.04 to 3.13) or that some/most/all friends drink (AOR 3.82, 95\% Cl 2.40 to 6.08); those given gentle reminders about taking care when drinking alcohol (AOR 1.82, 95\% Cl 1.15 to 2.88), practical advice (AOR 2.09, 95\% Cl 1.20 to 3.64) or designated time, led by the parent, to instil care around alcohol through a formal sit-down (AOR 1.79, 95\% Cl 1.07 to 2.99). The likelihood was reduced for parents aged $40-49$ years (AOR $0.52,95 \% \mathrm{Cl} 0.31$ to 0.89 ) and conversations providing information (AOR $0.53,95 \% \mathrm{Cl} 0.29$ to 0.98 ). Vomiting was significantly associated with some/most/all friends drinking alcohol (AOR 3.65, 95\% Cl 1.08 to 12.30), parent's beliefs about child's frequency of drinking alcohol (AOR 1.26, 95\% Cl 1.02 to 1.54), parental harmful/dependency drinking (AOR 3.75, 95\% Cl 1.13 to 12.50) and having a formal sitdown conversation (AOR 2.15, 95\% Cl 0.99 to 4.66). Conclusions We found evidence of mostly negative associations between young people's risk behaviours and different types of parent-child conversations. Conversations providing information were linked to a reduced tendency to have ever drunk alcohol. All other types of conversations were negatively associated with risk behaviours. Psychological reactance and conversation quality possibly explain these findings.
Strengths and limitations of this study

- A major strength of this study is the combination of two surveys, recruiting parents and their child, to identify the types of conversations they have had about drinking alcohol and its harmful consequences.

- Data included a large cross-sectional representative sample allowing for generalisability across the UK.

- The choice of the Alcohol Use Disorder Identification Test tool to assess parental drinking problems is a possible weakness, although it does allow for comparisons with other studies.

- The cross-sectional design of the study does not provide information about the temporal order of conversations and alcohol use, nor does it allow for inferences of cause-and-effect relationships.

- The self-select online survey, with parents gatekeeping child participation, has the potential for inducing bias because those who took part may be behaviourally and attitudinally different from those who did not, and this may have influenced our findings.

\section{BACKGROUND}

Young people in the UK typically first taste alcohol at the age of 13 years and levels of consumption tend to rapidly increase as they advance in age. ${ }^{12}$ Key findings from a 2016 national survey in the UK, ${ }^{2}$ the most recent data available, reported $53 \%$ of young people aged 13-17 years have drank alcohol at least once, and $11 \%$ will have drank in the last week, consuming an average of 9.6 units. Despite reducing trends in harmful drinking in the UK over the last 10 years, risky behaviours are still prevalent. ${ }^{3}$ Around 20\% of young people who drank alcohol in the last week got drunk, ${ }^{4}$ and in the 2015/20162017/18 period, there were 11610 hospital admissions for alcohol-related conditions in under 18 years. ${ }^{5}$ In the 'What about YOUth?' survey of 120000 young people aged 15 years 
in England, the most commonly reported consequences from drinking were doing something that young people later regretted $(15.7 \%)$ and hurting themselves $(8.6 \%){ }^{6}$ Early initiation of alcohol use and frequent drinking are also associated with adverse psychological, social and physical health consequences, such as initiation of drug use, suicide ideation, depression, school absenteeism and liver disease in later life. ${ }^{7-9}$

Health concerns about alcohol consumption in young people remain high. National guidelines state that young people aged 15-17 years should only consume alcohol under parental guidance and no more than once a week. ${ }^{10}$ Meanwhile, reducing the number of young people aged 11-15 years drinking alcohol and the amount they drink is key in the Alcohol Harm Reduction Strategy. ${ }^{11}$ In addition to legislation, alcohol use in young people is affected by a range of factors, including genetics, family and peer influences and also wider social and environmental contexts. $^{1012}$

In terms of family influences, there is an increasing awareness of the role that parents can have in the development of their children's drinking behaviour ${ }^{13-15}$; however, the various study findings are mixed. Sherriff and colleagues have explored the relationship between communication and supervision of alcohol consumption in the family. ${ }^{16-18}$ Their findings indicated that (1) parents engage in discussions with their child about drinking, (2) many need guidance to discuss 'sensible drinking' with young people, and (3) more information and support about young people and alcohol is needed. A key part of this study is to assess specific aspects of parent-child communication beyond that from the prior mentioned evidence. While there is no single or standardised definition of parent-child conversations or communication about alcohol, components of alcohol communication can be drawn from previous research and corroborated in studies undertaken since the 2016 Drinkaware Monitor Survey. Studies looking at parental strategies associated with reducing alcohol use have found the following to be effective: talking to and negotiating with children, parental monitoring, positive parent-child relationships, and communicating messages regarding health and consequences. ${ }^{19-23}$ In contrast, parental use of alcoholspecific communication, detailed and more frequent conversations, ${ }^{24} 25$ conversations about the negative consequences of alcohol use, ${ }^{26}$ and the introduction of alcohol use rules and discipline ${ }^{27}$ have all been associated with increased alcohol use in young people. However, these individual findings were either corroborated or contradicted in a 2017 meta-analysis of parenting and adolescent alcohol misuse. ${ }^{28}$ Neither alcohol-specific nor general communication was associated with initiation of alcohol use or misuse; reductions in the risk of alcohol use and misuse were associated with parental monitoring, better parent-child relationship quality and parental support. Such differing outcomes could be due to the type and level of communication offered and the quality of parent-child communication encounters. Reactance theory $^{29}$ could also offer another explanation as to why conversations and messages aimed at reducing alcohol use and harm may actually fail or even achieve the opposite; we will draw on this to help better understand our findings in the discussion.

In general, parents do talk to their children about alcohol, but current messages from the media, anecdotal experience (including cultural norms and family background) and research evidence about what and how to communicate can be contradictory and vary across cultures. ${ }^{28}$ Sawyer and colleagues explored parent-child communication in their qualitative study to identify the main types of alcohol-related conversations experienced by parents and their child. ${ }^{30}$ Conversation starters and topics were explored. The most salient and effective strategies to talk about harm reduction were deemed to be those where the opinions of both the parent and the child matched. Findings from the thematic analysis identified five distinguishable conversation types as follows: a gentle reminder (eg, care messages prior to a young person going to a party or event); formal sit-down (a designated time led by the parent to instil care around alcohol); triggered (initiated by an event of alcohol-related harm, either by the child or a fictional instance); practical advice (typically when alcohol use is expected and advice is provided on how to stay safe); and providing information (delivery of information about the effects of alcohol and possible dangers associated with alcohol).

The aim of this analysis was to investigate the potential associations between this range of parent-child conversation types and young people's (13-17 years) alcoholrelated risk behaviours. These conversation types will be explored alongside other known, or potential, risk and protective factors.

\section{METHODS}

\section{Study population}

This secondary analysis used data from the 2016 Drinkaware Monitor Survey ${ }^{2}$ on alcohol-related behaviours in a nationally representative sample of UK adults conducted between 25 November and 14 December 2016. ${ }^{2}$ The Drink Monitor Survey (DMS) sampled 1003 UK adults aged 28-80 years who also had at least one child aged 13-17 years. Sampling quotas were set based on the known population distribution of parents of this age group, and the final data were weighted to reflect this profile. The parent survey was conducted through the Ipsos MORI online panel, which consists of people who have signed up to take part in surveys, and an invitation was extended to their partners. Following the completion of the online survey, parents were asked for consent to survey their children about alcohol use and behaviours. If they were happy for their child to take part, they were then instructed to bring them to the computer, to read information about the child survey and to provide consent. Further instructions stated that the child should complete the online survey on their own and in private. A 
further 561 young people consented and took part. Data from the parent survey were then linked to the children's survey (matched pairs). The parent and child questionnaires were intentionally designed to overlap as much as possible (eg, 'Have you and/or your partner ever spoken to this child about drinking alcohol?' and 'Have your parents ever spoken to you about drinking alcohol?'). This created 'matched variables', therefore facilitating a comparison between child and parent responses. The final sample consisted of 561 parents and young people (total $\mathrm{n}=1122$ ). In order to minimise the level of missing data, the majority of questionnaire items were 'forced responses', meaning respondents must choose a response option in order to continue with the survey. Choices of 'don't know' or 'prefer not to answer' were frequently included as options. Details of the study design and the questionnaires used are published elsewhere. ${ }^{2}$

\section{Young people's alcohol-related risk behaviours}

Factors associated with young people's alcohol-related behaviours were based on exploring relationships with two variables from the child's survey': whether they had ever drunk an alcoholic drink (ie, a whole drink and not just a sip (no/yes) ) and ${ }^{2}$ whether they had experienced vomiting as a result of alcohol ingestion (no/yes). The first variable was identified as key through the literature, which suggests that age at first drink can determine later 'problem drinking'. ${ }^{31}$ This same question has been used in the Smoking, Drinking and Drug Use among Young People in England surveys ${ }^{1}$ and is recommended for use by researchers so that comparisons can be made across studies. The second variable, vomiting, was selected because the act itself can be harmful, but this measure is also a tangible event indicative of a level of intoxication that could increase exposure to other harmful consequences. Vomiting was also by far the highest reported type of harm in the child survey.

\section{Patient and public involvement}

There was no funding for this study or for patient and public involvement, and so we were unable to consult with young people and their parents for this piece of research.

\section{Factor variables}

Demographic variables: parent's employment status (employed full-time, employed part-time, unemployed, other, eg, student/retired); parent's age both continuous and broken down into groups (28-39, 40-49 and 50-72 years); young person's age (from 13 to 17 years); and young person's gender.

Parent's beliefs about their child's drinking: this was measured using the following three items: beliefs about how much their child drinks (I know a lot, I know a little); beliefs about how many of the child's friends the parent thinks drink alcohol (none/a few, some, most/all); and parent's beliefs how often their child drinks alcohol, a categorical variable treated as continuous $(0=$ only once or twice, $1=$ less than once/twice a year, $2=$ once or twice a year, $3=$ once every couple of months, $4=$ once a month, $5=$ two-three times a month, $6=$ once a week and $7=$ twothree times a week).

Parent's alcohol use: the Alcohol Use Disorder Identification Test (AUDIT) ${ }^{32}$ was completed by parents to measure drinking and potential harmful drinking among parents. The AUDIT comprises 10 questions measuring an individual's level of risk and/or harm in relation to alcohol consumption patterns. Each item carries a score of $0-4$, which gives an overall AUDIT score between 0 and 40 (zone 1: low risk (0-7), zone 2: hazardous, ${ }^{8-15}$ zone 3: harmful, zone 4 : dependency). The Cronbach's $\alpha$ for the scale was 0.73 in the DMS (compared with a mean of 0.8 in validation studies ${ }^{33}$ ). An additional question was also included to explore if the young person has ever seen the parent drunk: 'Has your child ever seen you drunk (by drunk we mean having drunk enough alcohol to be less in control, wobbly or under strong influence of alcohol or doing something or saying things you wouldn't normally say or do without drinking)?' (no/yes).

Conversations about alcohol: the DMS for parents asked 'Have you and/or your partner ever spoken to this child about drinking alcohol?' (question P18, no/yes). If yes, parents were asked two multiresponse questions listing possible types of conversations they may have had with their child about alcohol. These were questions 'Which, if any, of the below describe the type of conversations you/your partner have ever had with this child about drinking alcohol?' (question P19, five items excluding 'other' and 'don't know') and 'When you/ your partner have had conversations with this child about drinking alcohol, which of the following, if any, did you talk about?' (question P20, 14 items excluding other and don't know). The items were grouped into gentle reminder, formal sit-down, triggered, practical advice and providing information conversations (see table 1), which were consistent with the five types of parent-child communication identified in the literature. ${ }^{30}$ Corresponding factors were created to indicate whether or not that type of conversation had occurred at least once (no/yes).

\section{Data analysis}

Descriptive variables were either summarised using means $(\mathrm{m})$ and standard deviations (SD) or counts (n) and percentages $(\%)$. Separate models to describe the two risk behaviours were built using purposeful selection of variables. ${ }^{34}$ The first step was to use a series of bivariate logistic regressions to explore associations between the independent variables and the selected risk behaviour. Analyses were carried out on all available cases. Using the likelihood ratio (LR) test, any variables with a $\mathrm{p}$ value of $<0.25^{35}$ were selected as candidates for the multivariate model. Once created, all candidates were entered into a model simultaneously with the risk behaviour as the dependent variable. Young person's age and gender was added and maintained as a controlling variable regardless 
Table 1 Formulation of conversation-type factors from the DMS 2016

\begin{tabular}{|c|c|c|c|}
\hline $\begin{array}{l}\text { Conversation- } \\
\text { type factor }\end{array}$ & $\begin{array}{l}\text { Conversation-type } \\
\text { description }\end{array}$ & $\begin{array}{l}\text { DMS } 2016 \text { question } \\
\text { and item } ¥ \text { number }\end{array}$ & Item wording \\
\hline Gentle reminder & Gentle reminder & P19: 1 & $\begin{array}{l}\text { 1. Gentle reminders about the need to be careful about } \\
\text { drinking }\end{array}$ \\
\hline Formal sit-down & Formal sit-down & P19: 2 & $\begin{array}{l}\text { 2. Sitting them down for a detailed talk about the risks of } \\
\text { drinking and ways to avoid having problems related to drinking }\end{array}$ \\
\hline \multirow[t]{3}{*}{ Triggered } & \multirow[t]{3}{*}{$\begin{array}{l}\text { Triggered to have a } \\
\text { conversation }\end{array}$} & \multirow[t]{3}{*}{ P19: $3,4,5$} & $\begin{array}{l}\text { 3. Talking to them when you have discovered they have been } \\
\text { drinking }\end{array}$ \\
\hline & & & 4. Responding to questions they have asked about drinking \\
\hline & & & $\begin{array}{l}\text { 5. Discussing alcohol after something we have seen or heard } \\
\text { on the TV, radio, internet or in the street }\end{array}$ \\
\hline \multirow[t]{3}{*}{ Practical advice } & \multirow{3}{*}{$\begin{array}{l}\text { Behavioural } \\
\text { advice (topics of } \\
\text { conversation) }\end{array}$} & \multirow{3}{*}{$\begin{array}{l}\text { P20: } 1,2,3,9,10 \\
11\end{array}$} & 1. Ways to stay safe while drinking alcohol \\
\hline & & & 2. Ways to avoid getting too drunk \\
\hline & & & 11. How to say no when offered a drink \\
\hline \multirow{6}{*}{$\begin{array}{l}\text { Providing } \\
\text { information }\end{array}$} & \multirow{6}{*}{$\begin{array}{l}\text { Providing general } \\
\text { information/knowledge } \\
\text { about drinking alcohol } \\
\text { and its effects }\end{array}$} & \multirow{6}{*}{$\begin{array}{l}\text { P20: } 4,5,6,7,8 \\
12^{*}, 13,14\end{array}$} & 4. The importance of not losing control \\
\hline & & & 5. The risk of getting involved in violence \\
\hline & & & $\begin{array}{l}\text { 6. The short-term effects of drinking alcohol (such as feeling } \\
\text { unwell, being sick or dizzy) }\end{array}$ \\
\hline & & & 7. The risk of getting in trouble with the police \\
\hline & & & $\begin{array}{l}\text { 12.* The risks of unwise sexual activity as a result of drinking } \\
\text { too much }\end{array}$ \\
\hline & & & $\begin{array}{l}\text { 13. That some young people decide not to drink alcohol at } \\
\text { all }\end{array}$ \\
\hline
\end{tabular}

${ }^{*}$ Only asked of young people aged 16 years or over.

†All conversation-type factors were binary, with a yes indicating that at least one item was confirmed.

$\ddagger$ All items were binary (no/yes).

DMS, Drink Monitor Survey.

of significance. By using an iterative process of variable selection, independent variables were then removed if they were non-significant ( $p$ value $>0.1$ ) and not considered a confounder. Confounding was defined as a greater than $20 \%$ change in a coefficient in the nested model when compared with the full one. Using the final set of significant variables and confounders, variables originally not selected for the multivariate model are checked to see if they now make a significant (LR test $\mathrm{p}$ value $<0.1$ ) contribution. The model is iteratively reduced as before but only on the additional variables. The aim of this technique was to produce a parsimonious multivariate model of main effects for the risk behaviour variable. Effects are described in terms of adjusted ORs (AORs). STATA V.13 was used for all analyses.

\section{RESULTS}

There were a total of 561 cases of parent-child matched pairs available for analysis. Eight young people opted not to answer the question about whether or not they had drunk a whole drink of alcohol, so analyses related to this risk behaviour were based on a slightly reduced sample $(n=553)$.

Parents who took part in the survey were predominantly female $(62.4 \%)$, white British $(87.9 \%)$ compared with other black and minority ethnic groups (5.6\% Asian, $2.9 \%$ other white, $1.8 \%$ mixed ethnicity, $1.6 \%$ African/ Caribbean and $<1 \%$ other), had an average age of 43.7 years $(\mathrm{SD}=6.3)$, with approximately half of the sample stating they were Christian $(49.7 \%)$ and a large proportion stating no religion $(41 \%) ; 36.6 \%$ had a household income of less than $£ 30000,35.4 \% £ 30$ 000-50 000, $27.9 \% £ 50000+$. Young people who took part in the survey were on average 14.9 years old ( $\mathrm{SD}=1.4$ years), and there were slightly more boys $(52.0 \%)$ than girls $(48.0 \%)$. More detailed descriptive statistics of the independent variables by risk behaviour are displayed in table 2 .

\section{Young people's alcohol-related risk behaviours}

Of young people, $50.1 \%(\mathrm{n}=277)$ reported drinking a whole alcoholic drink (table 2), and $30 \%(n=83)$ of these were aged 13-14 years. Overall, just over a 10th (10.8\%, 
Table 2 Descriptive statistics of independent variables, by risk behaviours

Whether ever drank alcohol

\begin{tabular}{lll}
\hline No & & Yes \\
Count & Per cent & Count
\end{tabular}

$\begin{array}{llll}(n=276) & \% & (n=277) & \%\end{array}$

Whether experienced vomiting as a result of alcohol No Yes

Per cent Count Per cent Count

$(n=217) \quad \% \quad(n=60)$

Per cent

$\%$

Young person's age (years)

\begin{tabular}{|c|c|c|c|c|c|c|c|c|}
\hline 13 & 93 & 33.7 & 28 & 10.11 & 23 & 10.6 & 5 & 8.33 \\
\hline 14 & 65 & 23.55 & 55 & 19.86 & 48 & 22.12 & 7 & 11.67 \\
\hline 15 & 53 & 19.2 & 57 & 20.58 & 47 & 21.66 & 10 & 16.67 \\
\hline 17 & 23 & 8.33 & 69 & 24.91 & 45 & 20.74 & 24 & 40 \\
\hline $\begin{array}{l}\text { Young person's age } \\
\text { (continuous years) }\end{array}$ & $m=14.4$ & $\mathrm{SD}=1.31$ & $m=15.30$ & $\mathrm{SD}=1.32$ & $m=15.23$ & $\mathrm{SD}=1.30$ & $m=15.75$ & $S D=1.32$ \\
\hline Male & 145 & 52.54 & 142 & 51.26 & 119 & 54.84 & 23 & 38.33 \\
\hline Female & 131 & 47.46 & 135 & 48.74 & 98 & 45.16 & 37 & 61.67 \\
\hline \multicolumn{9}{|l|}{ Parent's age (years) } \\
\hline 28-39 & 63 & 22.83 & 69 & 24.91 & 47 & 21.66 & 22 & 36.67 \\
\hline
\end{tabular}

Employment status

\begin{tabular}{|c|c|c|c|c|c|c|c|c|}
\hline Employed Full-time & 137 & 49.64 & 157 & 56.68 & 124 & 57.14 & 33 & 55 \\
\hline Employed Part-time & 69 & 25 & 60 & 21.66 & 47 & 21.66 & 13 & 21.67 \\
\hline Unemployed & 15 & 5.43 & 24 & 8.66 & 16 & 7.37 & 8 & 13.33 \\
\hline \multicolumn{9}{|l|}{ AUDIT } \\
\hline Zone 1: low risk (0-7) & 198 & 71.74 & 161 & 58.12 & 133 & 61.29 & 28 & 46.67 \\
\hline $\begin{array}{l}\text { Zones } 3 \text { and } 4 \text { : } \\
\text { harmful (16-19) and } \\
\text { dependency }(20+)\end{array}$ & 27 & 9.78 & 38 & 13.72 & 26 & 11.98 & 12 & 20 \\
\hline \multicolumn{9}{|c|}{ Parent's belief about how much the child drinks } \\
\hline
\end{tabular}

Parent's belief about how many of the child's friends drink alcohol

\begin{tabular}{|c|c|c|c|c|c|c|c|c|}
\hline Most/all & 12 & 5.06 & 78 & 31.71 & 46 & 23.96 & 32 & 59.26 \\
\hline Some & 41 & 17.3 & 79 & 32.11 & 65 & 33.85 & 14 & 25.93 \\
\hline None/a few & 184 & 77.64 & 89 & 36.18 & 81 & 42.19 & 8 & 14.81 \\
\hline Only once or twice (0) & 4 & 57.14 & 48 & 21.72 & 42 & 24.28 & 6 & 12.5 \\
\hline Less often (1) & 0 & 0 & 12 & 5.43 & 10 & 5.78 & 2 & 4.17 \\
\hline $\begin{array}{l}\text { Once every couple of } \\
\text { months (3) }\end{array}$ & 0 & 0 & 31 & 14.03 & 26 & 15.03 & 5 & 10.42 \\
\hline
\end{tabular}

Continued 
Table 2 Continued

\begin{tabular}{|c|c|c|c|c|c|c|c|c|}
\hline & \multicolumn{4}{|c|}{ Whether ever drank alcohol } & \multicolumn{4}{|c|}{ Whether experienced vomiting as a result of alcohol } \\
\hline & \multicolumn{2}{|l|}{ No } & \multicolumn{2}{|l|}{ Yes } & \multicolumn{2}{|l|}{ No } & \multicolumn{2}{|l|}{ Yes } \\
\hline & Count & Per cent & Count & Per cent & Count & Per cent & Count & Per cent \\
\hline & $(n=276)$ & $\%$ & $(n=277)$ & $\%$ & $(n=217)$ & $\%$ & $(n=60)$ & $\%$ \\
\hline Once a month (4) & 0 & 0 & 25 & 11.31 & 18 & 10.4 & 7 & 14.58 \\
\hline 2-3 times a month (5) & 0 & 0 & 32 & 14.48 & 22 & 12.72 & 10 & 20.83 \\
\hline Once a week (6) & 0 & 0 & 17 & 7.69 & 6 & 3.47 & 11 & 22.92 \\
\hline 2-3 times a week (7) & 1 & 14.29 & 8 & 3.62 & 6 & 3.47 & 2 & 4.17 \\
\hline $\begin{array}{l}\text { Parent's belief about } \\
\text { how often the child } \\
\text { drinks (continuous) }\end{array}$ & $\mathrm{m}=1.57$ & $\mathrm{SD}=2.57$ & $\mathrm{~m}=2.80$ & $S D=2.08$ & $m=2.51$ & $\mathrm{SD}=1.98$ & $\mathrm{~m}=3.85$ & $\mathrm{SD}=2.11$ \\
\hline \multicolumn{9}{|c|}{ If the child has ever seen the parent drunk } \\
\hline Yes & 73 & 32.44 & 112 & 43.58 & 78 & 39.39 & 34 & 57.63 \\
\hline No & 152 & 67.56 & 145 & 56.42 & 120 & 60.61 & 25 & 42.37 \\
\hline \multicolumn{9}{|c|}{ Whether parent has ever spoken to the child about drinking alcohol } \\
\hline Yes & 225 & 83.96 & 254 & 92.7 & 197 & 91.63 & 57 & 96.61 \\
\hline No & 43 & 16.04 & 20 & 7.3 & 18 & 8.37 & 2 & 3.39 \\
\hline \multicolumn{9}{|c|}{ Whether had a gentle reminder conversation } \\
\hline No & 181 & 67.54 & 132 & 48.18 & 109 & 50.7 & 23 & 38.98 \\
\hline Yes & 87 & 32.46 & 142 & 51.82 & 106 & 49.3 & 36 & 61.02 \\
\hline \multicolumn{9}{|c|}{ Whether had a formal sit-down conversation } \\
\hline No & 216 & 80.6 & 182 & 66.42 & 149 & 69.3 & 33 & 55.93 \\
\hline Yes & 52 & 19.4 & 92 & 33.58 & 66 & 30.7 & 26 & 44.07 \\
\hline \multicolumn{9}{|c|}{ Whether had a triggered conversation } \\
\hline No & 106 & 39.55 & 94 & 34.31 & 77 & 35.81 & 17 & 28.81 \\
\hline Yes & 162 & 60.45 & 180 & 65.69 & 138 & 64.19 & 42 & 71.19 \\
\hline \multicolumn{9}{|c|}{ Whether they had a providing information conversation } \\
\hline No & 71 & 26.49 & 64 & 23.36 & 52 & 24.19 & 12 & 20.34 \\
\hline Yes & 197 & 73.51 & 210 & 76.64 & 163 & 75.81 & 47 & 79.66 \\
\hline \multicolumn{9}{|c|}{ Whether they had a practical advice conversation } \\
\hline No & 96 & 35.82 & 54 & 19.71 & 45 & 20.93 & 9 & 15.25 \\
\hline Yes & 172 & 64.18 & 220 & 80.29 & 170 & 79.07 & 50 & 84.75 \\
\hline
\end{tabular}

AUDIT, Alcohol Use Disorder Identification Test.

$\mathrm{n}=60$ ) of young people have experienced vomiting as a result of drinking alcohol. When constraining the sample to just those who reported drinking alcohol, this proportion rises to $21.7 \%$ (table 2 ).

\section{Parent's drinking behaviours}

Most parents $(89.7 \%)$ said that they drink alcohol and AUDIT scores indicated that almost two-thirds (64.9\%) are 'low-risk' drinkers, a quarter $(23.4 \%)$ were considered 'hazardous' drinkers, and small proportions carried out 'harmful' (4.6\%) or 'dependent' drinking behaviours $(7.1 \%)$. Of the total number of parents, $38.4 \%$ thought that their child had seen them drunk.

Most parents $(76.7 \%)$ were confident that they knew a lot about their child's drinking; $12.8 \%$ said they knew a little and only a small proportion $(10.5 \%)$ felt like they did not know anything.

\section{Parent-child conversations about alcohol}

Of the total number of parents, $84.0 \%$ whose child reported having ever drank and $91.6 \%$ whose child reported vomiting had a conversation with their child about alcohol. Overall, the most frequent conversations were providing information $(75.1 \%$ and $76.6 \%)$, practical advice $(72.3 \%$ and $80.3 \%)$ and externally triggered conversations $(63.1 \%$ and $65.7 \%)$ in those who had ever drank and experienced vomiting, respectively.

\section{Bivariate analyses}

Results of the bivariate logistic regression analyses for each risk behaviour are displayed in table 3 . 
Table 3 Bivariate logistic regression models of both risk behaviours

Whether ever drank alcohol

\section{Potential risk} factors
Young person's age (years)

\begin{tabular}{|l|}
13 \\
14 \\
15 \\
16 \\
17
\end{tabular}

Base

2.81

0.795

3.572

1.028

5.378

9.964

Young person's age (continuous)

1.675

$1.568<0.001 \quad 3.037$

\section{Lower}

$95 \% \mathrm{Cl}$ $P$ value

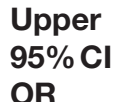

Whether experienced vomiting as a result of alcohol

Young person's gender

$\begin{array}{lcccccccccc}\text { Male } & \text { Base } & & & & & & & & & \\ \text { Female } & 1.052 & 0.179 & 0.765 & 0.754 & 1.469 & 1.953 & 0.583 & 0.025 & 1.088 & 3.507\end{array}$

Parent's age (years)

\begin{tabular}{|c|c|c|c|c|c|c|c|c|c|c|}
\hline 28-39 & Base & & & & & Base & & & & \\
\hline $40-49$ & 0.744 & 0.155 & 0.155 & 0.495 & 1.119 & 0.483 & 0.163 & 0.031 & 0.249 & 0.936 \\
\hline $50-72$ & 1.529 & 0.405 & 0.109 & 0.91 & 2.571 & 0.466 & 0.191 & 0.063 & 0.209 & 1.041 \\
\hline $\begin{array}{l}\text { Parent's age } \\
\text { (continuous) }\end{array}$ & 1.194 & 0.155 & 0.173 & 0.925 & 1.541 & 0.656 & 0.14 & 0.048 & 0.433 & 0.996 \\
\hline \multicolumn{11}{|c|}{ Employment status } \\
\hline $\begin{array}{l}\text { Employed } \\
\text { Full-time }\end{array}$ & Base & & & & & Base & & & & \\
\hline $\begin{array}{l}\text { Employed } \\
\text { Part-time }\end{array}$ & 0.759 & 0.161 & 0.192 & 0.501 & 1.149 & 1.039 & 0.384 & 0.917 & 0.504 & 2.144 \\
\hline Unemployed & 1.396 & 0.488 & 0.339 & 0.704 & 2.769 & 1.879 & 0.893 & 0.185 & 0.74 & 4.769 \\
\hline Other & 0.571 & 0.139 & 0.022 & 0.354 & 0.922 & 0.752 & 0.367 & 0.558 & 0.289 & 1.957 \\
\hline \multicolumn{11}{|l|}{ AUDIT } \\
\hline $\begin{array}{l}\text { Zone 1: low } \\
\text { risk (0-7) }\end{array}$ & Base & & & & & Base & & & & \\
\hline $\begin{array}{l}\text { Zone 2: } \\
\text { hazardous } \\
(8-15)\end{array}$ & 1.881 & 0.393 & 0.003 & 1.249 & 2.833 & 1.638 & 0.544 & 0.138 & 0.854 & 3.142 \\
\hline $\begin{array}{l}\text { Zones } 3 \text { and } \\
4: \text { harmful } \\
(16-19) \text { and } \\
\text { dependency } \\
(20+)\end{array}$ & 1.731 & 0.473 & 0.045 & 1.013 & 2.956 & 2.192 & 0.891 & 0.053 & 0.989 & 4.861 \\
\hline
\end{tabular}

Parent's beliefs about how much the child drinks

I know a lot. Base

Base

$\begin{array}{lllllllllll}\text { I know a little/l } & 1.562 & 0.319 & 0.029 & 1.047 & 2.33 & 2.17 & 0.669 & 0.012 & 1.186 & 3.971 \\ \text { don't know } \\ \text { anything. }\end{array}$

Parent's beliefs about how many of the child's friends drink alcohol

\begin{tabular}{lcccccccccr} 
None/a few & Base & \multicolumn{1}{c}{ Base } \\
Some & 3.984 & 0.923 & $<0.001$ & 2.529 & 6.274 & 2.181 & 1.032 & 0.1 & 0.862 & 5.516 \\
Most/all & 13.438 & 4.514 & $<0.001$ & 6.957 & 25.957 & 7.043 & 3.073 & $<0.001$ & 2.995 & 16.563
\end{tabular}

Parent's beliefs about how often the child drinks (continuous)

Continued 
Table 3 Continued

\begin{tabular}{|c|c|c|c|c|c|c|c|c|c|c|}
\hline \multirow[b]{2}{*}{$\begin{array}{l}\text { Potential risk } \\
\text { factors }\end{array}$} & \multicolumn{5}{|c|}{ Whether ever drank alcohol } & \multicolumn{5}{|c|}{$\begin{array}{l}\text { Whether experienced vomiting as a result of } \\
\text { alcohol }\end{array}$} \\
\hline & OR & SE & P value & $\begin{array}{l}\text { Lower } \\
95 \% \mathrm{CI} \\
\text { OR }\end{array}$ & $\begin{array}{l}\text { Upper } \\
95 \% \mathrm{Cl} \\
\text { OR }\end{array}$ & OR & SE & P-value & $\begin{array}{l}\text { Lower } \\
95 \% \mathrm{Cl} \\
\text { OR }\end{array}$ & $\begin{array}{l}\text { Upper } \\
95 \% \mathrm{CI} \\
\text { OR }\end{array}$ \\
\hline $\begin{array}{l}\text { Believed } \\
\text { drinking } \\
\text { frequency }\end{array}$ & 1.379 & 0.301 & 0.141 & 0.899 & 2.115 & 1.383 & 0.117 & $<0.001$ & 1.171 & 1.633 \\
\hline \multicolumn{11}{|c|}{ If the child has ever seen the parent drunk } \\
\hline No & Base & & & & & Base & & & & \\
\hline Yes & 1.608 & 0.306 & 0.012 & 1.108 & 2.334 & 2.092 & 0.63 & 0.014 & 1.16 & 3.774 \\
\hline \multicolumn{11}{|c|}{ Whether they had a gentle reminder conversation } \\
\hline No & Base & & & & & Base & & & & \\
\hline Yes & 2.238 & 0.398 & $<0.001$ & 1.579 & 3.172 & 1.61 & 0.482 & 0.112 & 0.894 & 2.896 \\
\hline \multicolumn{11}{|c|}{ Whether they had a formal sit-down conversation } \\
\hline No & Base & & & & & Base & & & & \\
\hline Yes & 2.1 & 0.421 & $<0.001$ & 1.417 & 3.111 & 1.779 & 0.535 & 0.056 & 0.986 & 3.209 \\
\hline \multicolumn{11}{|c|}{ Whether they had a triggered conversation } \\
\hline No & Base & & & & & Base & & & & \\
\hline Yes & 1.253 & 0.223 & 0.206 & 0.883 & 1.777 & 1.379 & 0.442 & 0.317 & 0.735 & 2.585 \\
\hline \multicolumn{11}{|c|}{ Whether they had a providing information conversation } \\
\hline No & Base & & & & & Base & & & & \\
\hline Yes & 1.183 & 0.235 & 0.399 & 0.801 & 1.746 & 1.249 & 0.45 & 0.537 & 0.616 & 2.533 \\
\hline \multicolumn{11}{|c|}{ Whether they had a practical advice conversation } \\
\hline No & Base & & & & & Base & & & & \\
\hline Yes & 2.274 & 0.451 & $<0.001$ & 1.542 & 3.354 & 1.471 & 0.587 & 0.334 & 0.673 & 3.215 \\
\hline
\end{tabular}

Constants estimated but not shown.

AUDIT, Alcohol Use Disorder Identification Test; Cl, Confidence Interval; OR, Odds Ratio; SE, Standard Error.

Ever drinking alcohol was significantly associated with the following variables: child's age, parent's AUDIT score, parent's beliefs about the amount their child drinks, parent's beliefs about how many of their child's friends drink, whether the children have seen their parent drunk and if they had a gentle reminder, formal sit-down or practical advice conversation. Experiencing vomiting was significantly associated with child's age, parent's age, AUDIT score, parent's beliefs about the amount their child drinks, parent's beliefs about how many of their child's friends drink alcohol, parent's beliefs of how often the child drinks, if the child has seen the parent drunk and if they had a formal sit-down conversation.

Statistically significant variables were used to create the initial pool of variables for the purposeful selection analysis.

\section{Multivariate analyses}

Table 4 displays the final model to describe which factors are associated with whether or not a young person had ever drunk alcohol. The increased likelihood of having ever drank alcohol was significantly associated with young person's age (OR=1.39, 95\% CI 1.17 to 1.66 , $\mathrm{p}$ value $<0.001)$; knowing a little about how much alcohol the young person drinks $(\mathrm{AOR}=1.80,95 \%$ CI 1.04 to $3.13, \mathrm{p}$ value $=0.036)$; if some $/ \mathrm{most} /$ all friends drink $(\mathrm{AOR}=3.82,95 \%$ CI 2.40 to 6.08 , $\mathrm{p}$ value $<0.001)$; providing a gentle reminder $(\mathrm{AOR}=1.82,95 \%$ CI 1.15 to 2.88 , $\mathrm{p}$ value $=0.010)$; having a practical advice conversation $(\mathrm{AOR}=2.09,95 \%$ CI 1.20 to 3.64 , $\mathrm{p}$ value $=0.009)$ and having a formal sit-down conversation $(\mathrm{AOR}=1.79$, 95\% CI 1.07 to $2.99, \mathrm{p}$ value 0.026 ). The reduced likelihood of having ever drank was significantly associated with parents aged $40-49$ years $(\mathrm{AOR}=0.52,95 \%$ CI 0.31 to 0.89 , $\mathrm{p}$ value $=0.018$ ) and having a conversation which provides information (AOR $=0.53,95 \%$ CI 0.29 to $0.98, \mathrm{p}$ value $=0.042$ ).

Table 5 displays the final model to describe which factors are associated with experiencing vomiting as a result of drinking alcohol. The increased likelihood of having ever drank alcohol was significantly associated with young person's age $(\mathrm{AOR}=1.44,95 \% \mathrm{CI} 1.02$ to 2.02, $\mathrm{p}$ value $=0.037)$; being a young female $(\mathrm{AOR}=3.71$, $95 \%$ CI 1.64 to 8.37 , $\mathrm{p}$ value $=0.002)$, if some $/ \mathrm{most} /$ all friends drink alcohol (AOR=3.65, 95\% CI 1.08 to 12.30, $\mathrm{p}$ value $=0.037$ ); parent's beliefs of how often their child 
Table 4 Final multivariate logistic regression model of factors associated with whether a young person has ever drunk alcohol

\begin{tabular}{|c|c|c|c|c|}
\hline $\begin{array}{l}\text { Whether young } \\
\text { person has ever } \\
\text { drank alcohol }\end{array}$ & AOR & SE & P value* & $\begin{array}{l}95 \% \mathrm{Cl} \\
\text { AOR }\end{array}$ \\
\hline $\begin{array}{l}\text { Young person's } \\
\text { age }\end{array}$ & 1.39 & 0.12 & $<0.001$ & 1.17 to 1.66 \\
\hline \multicolumn{5}{|c|}{ Young person's gender } \\
\hline Male & Base & & & \\
\hline Female & 0.95 & 0.21 & 0.808 & 0.61 to 1.46 \\
\hline \multicolumn{5}{|l|}{ Parent's age } \\
\hline 28-39 & Base & & & \\
\hline $40-49$ & 0.52 & 0.14 & 0.018 & 0.31 to 0.89 \\
\hline $50-72$ & 1.17 & 0.41 & 0.649 & 0.59 to 2.31 \\
\hline \multicolumn{5}{|l|}{ Employment status } \\
\hline \multicolumn{5}{|c|}{$\begin{array}{l}\text { Employed Full- Base } \\
\text { time }\end{array}$} \\
\hline $\begin{array}{l}\text { Employed } \\
\text { Part-time }\end{array}$ & 0.86 & 0.24 & 0.588 & 0.50 to 1.47 \\
\hline Unemployed & 2.16 & 1.07 & 0.12 & 0.82 to 5.72 \\
\hline Other & 0.64 & 0.2 & 0.164 & 0.35 to 1.20 \\
\hline
\end{tabular}

Parent's beliefs about how much the child drinks

$\begin{array}{lrrrr}\text { Know a lot } & \text { Base } & & & \\ \text { Know a little } & 1.8 & 0.51 & 0.036 & 1.04 \text { to } 3.13\end{array}$

Parent's beliefs about how many of the child's friends drink alcohol

\begin{tabular}{|c|c|c|c|c|}
\hline None/a few & Base & & & \\
\hline Some/most/all & 3.82 & 0.91 & $<0.001$ & 2.40 to 6.08 \\
\hline \multicolumn{5}{|c|}{ Whether they had a gentle reminder } \\
\hline No & Base & & & \\
\hline Yes & 1.82 & 0.42 & 0.01 & 1.15 to 2.88 \\
\hline \multicolumn{5}{|c|}{ Whether they had a practical advice conversation } \\
\hline No & Base & & & \\
\hline Yes & 2.09 & 0.59 & 0.009 & 1.20 to 3.64 \\
\hline \multicolumn{5}{|c|}{ Whether they provided information } \\
\hline No & Base & & & \\
\hline Yes & 0.53 & 0.16 & 0.042 & 0.29 to 0.98 \\
\hline \multicolumn{5}{|c|}{ Whether they had formal sit-down } \\
\hline No & Base & & & \\
\hline Yes & 1.79 & 0.47 & 0.026 & 1.07 to 2.99 \\
\hline
\end{tabular}

Analysis was performed using purposeful selection and multivariate logistic regression analysis.

*Significant results are bolded; constant estimated but not shown AOR, adjusted OR; CI, Confidence Interval; SE, Standard Error.

drinks $(\mathrm{AOR}=1.26,95 \%$ CI 1.02 to 1.54 , $\mathrm{p}$ value $=0.029)$; and harmful and dependency parental alcohol use (AOR=3.75, 95\% CI 1.13 to 12.50 , p value=0.031). Additionally, whether parents had a formal sit-down conversation with their child was just above the $5 \%$
Table 5 Final multivariate logistic regression model of factors associated with whether a young person has experienced vomiting as a result of alcohol

\begin{tabular}{|c|c|c|c|c|}
\hline $\begin{array}{l}\text { Whether young } \\
\text { person has } \\
\text { experienced } \\
\text { vomiting }\end{array}$ & AOR & SE & P value* & $95 \% \mathrm{Cl}$ AOR \\
\hline $\begin{array}{l}\text { Young person's } \\
\text { age }\end{array}$ & 1.44 & 0.25 & 0.037 & 1.02 to 2.02 \\
\hline \multicolumn{5}{|c|}{ Young person's gender } \\
\hline Male & Base & & & \\
\hline Female & 3.71 & 1.54 & 0.002 & 1.64 to 8.37 \\
\hline \multicolumn{5}{|c|}{$\begin{array}{l}\text { Parent's beliefs about how many of the child's friends drink } \\
\text { alcohol }\end{array}$} \\
\hline None/a few & Base & & & \\
\hline Some/most/all & 3.65 & 2.261 & 0.037 & 1.08 to 12.30 \\
\hline $\begin{array}{l}\text { Parent's beliefs } \\
\text { about how often } \\
\text { the child drinks } \\
\text { (continuous) }\end{array}$ & 1.26 & 0.13 & 0.029 & 1.02 to 1.54 \\
\hline \multicolumn{5}{|l|}{ AUDIT score } \\
\hline $\begin{array}{l}\text { Zone } 1 \text { : low } \\
\text { risk }(0-7)\end{array}$ & Base & & & \\
\hline $\begin{array}{l}\text { Zone 2: } \\
\text { hazardous } \\
(8-15)\end{array}$ & 2.24 & 0.993 & 0.07 & 0.94 to 5.34 \\
\hline $\begin{array}{l}\text { Zones } 3 \text { and 4: } \\
\text { harmful (16-19) } \\
\text { and dependency } \\
(20+)\end{array}$ & 3.75 & 2.303 & 0.031 & 1.13 to 12.50 \\
\hline \multicolumn{5}{|c|}{ Whether they had a formal sit-down } \\
\hline No & Base & & & \\
\hline Yes & 2.15 & 0.848 & 0.052 & 0.99 to 4.66 \\
\hline
\end{tabular}

Analysis performed using purposeful selection of variables andmultivariate logistic regression analysis.

*Significant results are bolded; constant estimated but not shown. AOR, adjusted odds ratio; AUDIT, Alcohol Use Disorder Identification Test; Cl, Confidence Interval; SE, Standard Error.

cut-off for significance but also had a positive association $(\mathrm{AOR}=2.15,95 \%$ CI 0.99 to 4.66, $\mathrm{p}$ value $=0.052)$. An LR test comparing this final model with and without the formal sit-down factor results in $p$ value $=0.0508$.

\section{DISCUSSION}

Our cross-sectional findings, from a matched sample of parents and children, highlight unique and mostly negative links between several types of conversations and first alcohol consumption (ever having had a drink) and harm (vomiting), after adjustment for age and gender. All forms of conversations, except for those providing information, were associated with an increased probability of ever having had a drink. However, only formal sit-down conversations were linked to harmful behaviour 
(although on the margin of statistical significance), and this association was negative.

Our findings need to be considered within the context of increasing age being associated with drinking alcohol and having a first drink; these trends have been observed in many countries including the UK ${ }^{36}{ }^{37}$ In addition, although relevant for a number of countries, a recent WHO report ${ }^{36}$ singles out the UK for the largest reduction of weekly alcohol consumption among young people between 2002 and 2014. A number of explanations have been explored, but without success. Interestingly, inequalities are not linked to alcohol-related risks as usually expected; young people from affluent families are more likely to be at risk and are more likely to drink weekly. ${ }^{36}$ Thus, alcohol consumption behaviour is at variance to many other health risk behaviours which tend to show a strong social gradient, with more disadvantaged groups being more at risk. From a public health perspective, this reduction is very welcome, but in the UK, harm due to alcohol use is still likely in almost $20 \%$ of older adolescents. These figures are very similar to ours, with close to $20 \%$ reporting alcohol-related harm (vomiting) in our 16 and 17-year-old participants compared with only $5 \%$ of young people aged 13 and 14 years.

As already indicated, alcohol-related risk behaviours increase with age, but likewise, the likelihood of conversations, as reported by parents, increases for older children. ${ }^{48}$ In our study, close to $90 \%$ of all parents reported to have spoken to their child about alcohol; children report similar figures. Overall, the proportions rise with age, but not dramatically; $78 \%$ of young people aged 13 years report to have been spoken to by their parents. ${ }^{2}$ It is likely that alcohol use and conversations, at least at a granular level, take place at around the same time, with actual or expected alcohol use, for example, at a party, triggering conversations. ${ }^{30}$

In a recent systematic review of parental attitudes and children's alcohol consumption, ${ }^{39}$ findings suggest that parental attitudes, norms and beliefs about child behaviour, combined with conversations, shapes their own child's behaviour. However, child behaviour, in particular, vomiting, may shape parental attitudes (by raising concern or, alternatively, becoming more accepting ${ }^{40}$ ) and behaviour. We therefore need to consider how our findings could relate to these two opposing pathways of (1) conversations between parent and child shaping child behaviour and (2) alcohol-related risk behaviour triggering and shaping conversations.

Our data show that both risk behaviours are rare in younger age children but increase rapidly with age. In parallel, peers become more important, while parental influence tends to reduce. Given that conversations, except for those providing information, are associated with a higher probability of ever drinking alcohol and that parents are unlikely to encourage drinking explicitly, negative associations may possibly be explained by (1) the quality of conversations, (2) implicit messages being sent out or (3) raising interest in those who have not yet had a first drink. Conversations could inadvertently trigger curiosity of trying alcohol, either on a first occasion or testing out 'one's limits' around drunkenness. Sharmin et $a t^{40}$ focused on risky drinking (consuming $\geq 5$ standard drinks on a single occasion at least monthly) and found that perceived parental approval of drinking alcohol was linked to higher levels of risky drinking, but the reverse did not apply; that is, perceived disapproval was not associated with lower levels of risky drinking.

The reverse pathway of child behaviour triggering conversations has been discussed by Sawyer $e t a l^{30}$; conversation starters include the child going to a party or a family member getting drunk at a family celebration. This suggests that conversations can be context dependent and in response to a planned attendance to a social event, or plain parental curiosity.

This interplay between changes in child behaviour, and parental action can be explored from the perspective of psychological reactance theory. ${ }^{29}$ This theory helps to explain why campaigns intended to promote health, for example, binge drinking reduction among college students, can in effect increase the probability of the behaviours which they were supposed to discourage. ${ }^{41}$ Applied to health promotion messages, the theory suggests that such messages may be perceived as restricting or threatening freedom, thereby eliciting health-impairing behaviours expressive of freedom. ${ }^{42}$ In particular, sit-down conversations may be experienced by children as heavy-handed efforts to restrict what the child is allowed to do; this could be a possible explanation for the substantive negative association with both risk behaviours. Similarly, practical guidance conversations, which are negatively associated with a first drink, may trigger reactance. Only conversations providing information are associated with a lower risk of having had a first drink. Similarly, Sawyer et a $\vec{P}^{0}$ suggested that children are not keen on sit-down conversations; instead they prefer more informal conversations.

The complexity of the pattern around conversations and child alcohol use also applies to parental beliefs about their child's alcohol use, with the possibility of bidirectional effects playing out during the child's development within their family context. Knowing only a little about how much their child drinks was associated with a higher chance of alcohol use. On the other hand, parental beliefs around drinking frequency were linked to the experience of vomiting in young people. These opposing relationships between parental beliefs and behavioural risks may be due to more frequent drinking in those who report having vomited. In contrast, having a drink is positively associated with the parent's beliefs about how much the child drinks, which may be linked to parental monitoring; children of parents who believe they know little or nothing are more likely to have had a first whole drink. These findings are in line with the literature on the risk-reducing role of monitoring in relation to alcohol use in young people. ${ }^{22} 23$

The progression from having a drink, possibly in the presence of a parent or other adults, to being sick due to 
alcohol, already at a young age, has been highlighted in the literature. The item 'Sitting them down for a detailed talk about the risks of drinking, and ways to avoid having problems related to drinking' describes a clearly defined activity and form of interaction, but does not provide any information about the motive or trigger. Liberal parenting may include positive attitudes towards alcohol and the provision of alcohol (eg, allowing sips to underage young people or providing alcohol for parties) or it could be in response to an issue where the parents are concerned about alcohol consumption by their child. Pursuing the former line of argument of liberal parenting, that is, permitting the consumption of alcohol, carries the risk of the young person considering the consumption of alcohol as socially acceptable. This pattern of development has been identified in research; prospective studies have shown that such behaviour is linked to early drinking and/or alcohol misuse in later life. Parents and policy makers have therefore been recommended to promote avoiding even a sip, ${ }^{28}$ particularly in Anglo-Saxon and north European countries where this link has been identified, in contrast to south European countries, for example.

Finally, our data indicated links between alcohol-related risk in young people and social factors such as peer drinking and parental risk of alcohol dependency and harm. Increased probabilities of both risk behaviours were associated with peer drinking in young people, while higher parental AUDIT scores appeared to be linked to reports of vomiting. Social learning theory ${ }^{43}$ proposes mechanisms, that is, learning by observation and the influence of role models or significant others, which may explain the effect of peers ${ }^{44}$ and parents and their alcohol use. ${ }^{45}$ These social influences appear to be important factors especially in relation to vomiting (see the highly significant association with AUDIT scores). In addition, peer behaviour tends to create descriptive norms which permit and dictate alcohol use. This is in line with a body of evidence that demonstrates the increased influence of peers, social norms and peer selection at this life stage. ${ }^{46-50}$

\section{Strength and weaknesses}

A major strength of this cross-sectional survey is the fairly unique combination of linked parent and child surveys, whereby parents and their child have been recruited into the study and questioned on the type of conversations they have; we are not aware of another UK-representative study in this regard. Recruiting such samples presents a considerable challenge as is well known in the field of dyadic and relationship research. ${ }^{51}$ Importantly, the sample was carefully recruited, is representative of this segment of the UK population and therefore allows for generalisation; the size of the sample (561 parent-child pairs) was sufficient to carry out statistical modelling of two key risk behaviours.

The main limitation of this study is the cross-sectional nature of the survey, which does not allow for the identification of the temporal order of reported events and causality. This issue has been raised in systematic reviews ${ }^{52} 53$ and intervention studies. ${ }^{54}$ However, as the survey presented here is one of the first to include parents and children from the same family, we consider this weakness to be acceptable.

Only a 10th of the entire sample of young people experienced vomiting as a result of drinking alcohol, and of those, the majority $(62 \%)$ were female. The over-representation of this subgroup is a limitation of the data, and so interpretation of this primary negative consequence should be carried out with this in mind.

The choice of questions and the AUDIT alcohol risk questionnaire was carefully planned and is in line with many other surveys, a strong point, but the psychometric properties of some of the newly developed questions should be further scrutinised. The survey captured the nature of conversations and addressed occurrence in a binary way; comparisons with studies focusing on the frequency and quality of conversations were not possible. The widely used AUDIT questionnaire has been developed for clinical and addiction screening purposes, ${ }^{32}$ and its usefulness for population-based studies has been questioned.

The survey is only available to those who have self-selected to sign up to be a part of the Ipsos MORI online panel and who meet the sampling criteria. Parents then act as gatekeepers to the participation of young people. Therefore, parents who had consented for their child to take part in the survey may have been attitudinally or behaviourally different from those who had not. This could mean that responses may not be representative of the UK population. However, analyses into the potential systematic differences between those who had and had not consented to the survey found little evidence to support this. ${ }^{2}$

\section{CONCLUSIONS AND IMPLICATIONS}

This exploratory, multivariate analysis of a nationally representative dataset of matched parent and child pairs provides some surprising results. In terms of risk factors, conversations are mostly negatively associated with having had a first drink with the exception of conversations providing information. Alcohol harm is negatively associated with sit-down conversations. Our detailed discussion of possible explanations of these findings indicated the possible role of reactance, but also the possibility that conversations may raise awareness and trigger curiosity to try out alcohol and for the child testing their limits.

Our discussion highlighted the complexity of the nature, motives and triggers of conversations between parents and children, and of alcohol use, and all of this entangled in the intricacies of the young person's development, family and wider social contexts. In addition, the findings need to be seen in the context of a reduction of alcohol use in young people, with the UK having seen one of the biggest reductions in the last decade or so, although more in boys than in girls.

The findings, rather than providing strong pointers for public health and health promotion practitioners, point to challenges which would need to be addressed in future research. Similar conclusions were reached in a recent Cochrane review on family interventions which showed 
minimal effects and some even harmful. ${ }^{54}$ The quality of conversations and parent-child connectedness (quality of relationship) are likely targets for further research.

Finally, conversations about alcohol should be considered conducted by parents in the context of other potentially harmful behaviours which can be sensitive; this includes topics such as smoking, drug use and sexual behaviour. ${ }^{55} 56$ Future studies should address how to approach and conduct conversations around these other topics, and identify which common factors help to reduce risks to young people's health.

\section{Twitter Anna-Marie Jones @Anna_MarieJones}

Acknowledgements We are grateful to The Drinkaware Trust, in particular, $\mathrm{Dr}$ John, for providing access to the Drinkaware Monitor Survey data and for their support in responding to queries regarding the methodology and questionnaire design.

Contributors Authors' contributions: NS, AS, JWH, ND and LC conceptualised the research study. A-MJ designed and conducted the analysis. All authors contributed, reviewed and approved the final manuscript.

Funding The authors have not declared a specific grant for this research from any funding agency in the public, commercial or not-for-profit sectors.

Competing interests NS has previously received research funding from The Drinkaware Trust. No authors received funding for any aspect of this manuscript, the data for which was requested from The Drinkaware Trust as part of an open data sharing agreement.

Patient and public involvement Patients and/or the public were not involved in the design, conduct, reporting or dissemination plans of this research.

Patient consent for publication Not applicable.

Ethics approval As per University of Brighton's research governance guidelines, ethical approval was not required for the secondary analysis of this dataset as (1) access to and use of the data are managed by an external organisation that complies with all legal requirements concerning data protection and (2) the data are not sensitive and the dataset is fully anonymised.

Provenance and peer review Not commissioned; externally peer reviewed.

Data availability statement Data may be obtained from a third party and are not publicly available. The authors are not the data custodians and do not have permission to share the dataset. The dataset used for this analysis is owned by the Drinkaware Trust. Data may be requested directly from the Drinkaware Trust by emailing research@drinkaware.co.uk.

Open access This is an open access article distributed in accordance with the Creative Commons Attribution Non Commercial (CC BY-NC 4.0) license, which permits others to distribute, remix, adapt, build upon this work non-commercially, and license their derivative works on different terms, provided the original work is properly cited, appropriate credit is given, any changes made indicated, and the use is non-commercial. See: http://creativecommons.org/licenses/by-nc/4.0/.

\section{ORCID iD}

Anna-Marie Jones http://orcid.org/0000-0002-4126-1915

\section{REFERENCES}

1 Fuller E. Smoking drinking and drug use among young people in England in 2014. Leeds: the health and social care information centre, 2015. Available: https://digital.nhs.uk/data-and-information/ publications/statistical/smoking-drinking-and-drug-use-amongyoung-people-in-england/2014

2 Adkins L. Teenage drinking and the role of parents and guardians: findings from Drinkaware monitor 2016. London: Ipsos-MORI, 2017.

3 Public Health England. Young people are less likely to drink; does that mean it isn't a problem?2016. Available: https:// publichealthmatters.blog.gov.uk/2016/08/02/young-people-are-lesslikely-to-drink-does-that-mean-it-isnt-a-problem/

4 National Statistics. Smoking, drinking and drug use among young people in England - 2016, 2017. Available: https://digital.nhs.uk/ data-and-information/publications/statistical/smoking-drinking-anddrug-use-among-young-people-in-england/2016

5 Public Health England. Local alcohol profiles for England, 2018. Available: https://fingertips.phe.org.uk/profile/local-alcohol-profiles/ data\#page/3/gid/1938132984/pat/6/par/E12000004/ati/102/are/ E06000015/iid/92904/age/173/sex/4

6 Health and Social Care Information Centre. Health and Wellbeing of 15-year-olds in England - Main findings from the What About YOUth? Survey 2014. London: Health and Social Care Information Centre, 2015. https://data.london.gov.uk/dataset/health-and-wellbeing-of15-year-olds-what-about-youth-survey-borough

7 Bellis MA, Phillips-Howard PA, Hughes $\mathrm{K}$, et al. Teenage drinking, alcohol availability and pricing: a cross-sectional study of risk and protective factors for alcohol-related harms in school children. BMC Public Health 2009;9:380.

8 Swahn MH, Bossarte RM, Gender BRM. Gender, early alcohol use, and suicide ideation and attempts: findings from the 2005 youth risk behavior survey. J Adolesc Health 2007;41:175-81.

9 Miller JW, Naimi TS, Brewer RD, et al. Binge drinking and associated health risk behaviors among high school students. Pediatrics 2007;119:76-85.

10 Donaldson L. Health guidance on the consumption of alcohol by chioldren and young people do. UK: Department of Health, 2009.

11 Department of Health.. 2010 to 2015 government policy: harmful drinking. London: Department of Health; 2015. Available: https:// www.gov.uk/government/publications/2010-to-2015-governmentpolicy-harmful-drinking/2010-to-2015-government-policy-harmfuldrinking.

12 Schuckit MA. An overview of genetic influences in alcoholism. $J$ Subst Abuse Treat 2009;36:S1-14.

13 Jacob N, MacArthur GJ, Hickman M, et al. A qualitative investigation of the role of the family in structuring young people's alcohol use. Eur $J$ Public Health 2016;26:102-10.

14 Valentine G, Jayne M, Gould M. The proximity effect: The role of the affective space of family life in shaping children's knowledge about alcohol and its social and health implications. Childhood 2014;21:103-18.

15 Valentine G, Harris CH, Evaluation TC. Final report for Drinkaware, 2016. Available: https://www.drinkaware.co.uk/research/ourresearch-and-evaluation-reports/evaluation-of-online-underageguidance/

16 Sherriff N, Cox L, Coleman L, et al. Communication and supervision of alcohol in the family: parental perspectives. Child Soc 2008:22:370-82.

17 Cox L, Sherriff N, Coleman LM, et al. Parent-young people communication about alcohol. final report to. Brighton: Alcohol Education and Research Council (AERC), 2006.

18 Sherriff N, Cox L, Coleman LM. Sensible drinking? talking about alcohol.Next generation. , 2006: 2, 14-15.

19 Highet G. Young people talking about how parents respond to their use of788 these two drugs AU - Highet. Drugs 2005;12:113-24.

20 Cohen DA, Richardson J, LaBree L. Parenting behaviors and the onset of smoking and alcohol use: a longitudinal study. Pediatrics 1994:94:368-75.

21 Reimuller A, Hussong A, Ennett ST. The influence of alcohol-specific communication on adolescent alcohol use and alcohol-related consequences. Prev Sci 2011;12:389-400.

22 Barnes GM, Hoffman JH, Welte JW, et al. Effects of parental monitoring and peer deviance on substance use and delinquency. $J$ Marriage Fam 2006;68:1084-104.

23 Lipperman-Kreda S, Gruenewald PJ, Bersamin M, et al. Adolescent drinking in different contexts: what behaviors do parents control? Addict Behav Rep 2017;6:39-44.

24 Carver H, Elliott L, Kennedy C, et al. Parent-child connectedness and communication in relation to alcohol, tobacco and drug use in adolescence: an integrative review of the literature. Drugs: Education, Prevention \& Policy 2017;24:119-33.

25 Van Der Vorst H, Burk WJ, Engels RCME. The role of parental alcohol-specific communication in early adolescents' alcohol use. Drug Alcohol Depend 2010;111:183-90.

26 Boyle JR, Boekeloo BO. The association between parent communication and College freshmen's alcohol use. J Drug Educ 2009:39:113-31.

27 Ennett ST, Bauman KE, Foshee VA, et al. Parent-Child communication about adolescent tobacco and alcohol use: what do parents say and does it affect youth behavior? J Marriage Fam 2001;63:48-62.

28 Yap MBH, Cheong TWK, Zaravinos-Tsakos F, et al. Modifiable parenting factors associated with adolescent alcohol misuse: a systematic review and meta-analysis of longitudinal studies. Addiction 2017;112:1142-62. 
29 Brehm JW. A theory of psychological reactance. Oxford England: Academic Press, 1966.

30 Sawyer A, Coleman L, Cooke R, et al. Understanding conversations about alcohol between parents and their $15-17$ year olds: a qualitative study. BMC Public Health 2018;18:631.

31 Liang W, Chikritzhs T. Age at first use of alcohol predicts the risk of heavy alcohol use in early adulthood: a longitudinal study in the United States. Int J Drug Policy 2015;26:131-4.

32 Saunders JB, Aasland OG, Babor TF, et al. Development of the alcohol use disorders identification test (AUDIT): WHO Collaborative project on early detection of persons with harmful alcohol consumption--II. Addiction 1993;88:791-804.

33 Cd M-G, Zuardi AW, Loureiro SR, et al. Alcohol use disorders identification test (audit): an updated systematic review of psychometric properties. Psychology \& Neuroscience 2009;2:83-97.

34 Hosmer DW, Lemeshow S. Applied logistic regression. 2nd. New York: Wiley and Sons, 2000.

35 Bursac Z, Gauss CH, Williams DK, et al. Purposeful selection of variables in logistic regression. Source Code Biol Med 2008;3:17.

36 Inchley J, Currie D, Vieno A, et al. Adolescent alcohol-related behaviours: trends and inequalities in the who European region, 2002-2014. Copenhagen: WHO Regional Office for Europe, 2018.

37 Oldham M, Holmes J, Whitaker V, et al. Youth drinking in decline. Available: http://eprints.whiterose.ac.uk/136587/

38 NatCen social research UCL. Health service for England 2016 , children's health. NHS Digital, 2017.

39 Tael-Öeren M, Naughton F, Sutton S. The relationship between parental attitudes and children's alcohol use: a systematic review and meta-analysis. Addiction 2019;114:1527-46.

40 Sharmin S, Kypri K, Khanam M, et al. Effects of parental alcohol rules on risky drinking and related problems in adolescence: systematic review and meta-analysis. Drug Alcohol Depend 2017;178:243-56.

41 Richards AS, Banas JA. Inoculating against reactance to persuasive health messages. Health Commun 2015;30:451-60.

42 Brehm SS, Brehm JW. Psychological Reactance: a theory of freedom and control. New York: Academic Press, 1981.

43 Bandura A. Social learning theory. Prentice-Hall, 1977: 1-247.

44 Schuler MS, Tucker JS, Pedersen ER, et al. Relative influence of perceived peer and family substance use on adolescent alcohol, cigarette, and marijuana use across middle and high school. Addict Behav 2019:88:99-105.

45 Mahedy L, MacArthur GJ, Hammerton G, et al. The effect of parental drinking on alcohol use in young adults: the mediating role of parental monitoring and peer deviance. Addiction 2018;113:2041-50.

46 Jackson KM, Roberts ME, Colby SM, et al. Willingness to drink as a function of peer offers and peer norms in early adolescence. $J$ Stud Alcohol Drugs 2014;75:404-14.

47 Moore GF, Littlecott HJ, School-, et al. And health behaviors: multilevel analysis of a national survey in Wales, United Kingdom. $J$ School Health 2015;85:267-75.

48 Mundt MP, Mercken L, Zakletskaia L. Peer selection and influence effects on adolescent alcohol use: a stochastic actor-based model. BMC Pediatr 2012;12:115.

49 Grigsby TJ, Forster M, Unger JB, et al. Predictors of alcoholrelated negative consequences in adolescents: a systematic review of the literature and implications for future research. J Adolesc 2016;48:18-35

50 Carrus G, Panno A, Deiana L, et al. Group membership and adolescents' alcohol intake: the role of drinking motives. J Stud Alcohol Drugs 2016;77:143-9.

51 Ummel D, Achille M. How not to let secrets out when conducting qualitative research with dyads. Qual Health Res 2016;26:807-15.

52 Visser L, de Winter AF, Reijneveld SA. The parent-child relationship and adolescent alcohol use: a systematic review of longitudinal studies. BMC Public Health 2012;12:886.

53 Meque I, Salom C, Betts KS, et al. Predictors of alcohol use disorders among young adults: a systematic review of longitudinal studies. Alcohol Alcohol 2019;54:310-24.

54 Gilligan C, Wolfenden L, Foxcroft DR, et al. Family-Based prevention programmes for alcohol use in young people. Cochrane Database Syst Rev 2019;3:CD012287.

55 National Society for the Prevention of Cruelty to Children. Talking about difficult topics: NSPCC;, 2019. Available: https://www.nspcc. org.uk/preventing-abuse/keeping-children-safe/talking-aboutdifficult-topics/

56 FRANK. Worried about a child: Frank, 2019. Available: https://www. talktofrank.com/get-help/worried-about-a-child\#helping-them-openup-about-drugs-and-alcohol 